\title{
Mcl-1-dependent activation of Beclin 1 mediates autophagic cell death induced by sorafenib and SC-59 in hepatocellular carcinoma cells
}

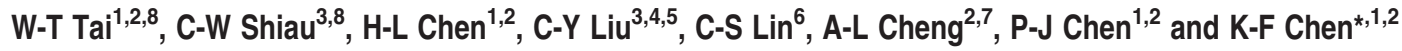

We investigated the molecular mechanisms underlying the effect of sorafenib and SC-59, a novel sorafenib derivative, on hepatocellular carcinoma (HCC). Sorafenib activated autophagy in a dose- and time-dependent manner in the HCC cell lines PLC5, Sk-Hep1, HepG2 and Hep3B. Sorafenib downregulated phospho-STAT3 (P-STAT3) and subsequently reduced the expression of myeloid cell leukemia-1 (Mcl-1). Inhibition of Mcl-1 by sorafenib resulted in disruption of the Beclin 1-Mcl-1 complex; however, sorafenib did not affect the amount of Beclin 1, suggesting that sorafenib treatment released Beclin 1 from binding with Mcl-1. Silencing of SHP-1 by small interference RNA (siRNA) reduced the effect of sorafenib on P-STAT3 and autophagy. Ectopic expression of Mcl-1 abolished the effect of sorafenib on autophagy. Knockdown of Beclin 1 by siRNA protected the cells from sorafenib-induced autophagy. Moreover, SC-59, a sorafenib derivative, had a more potent effect on cancer cell viability than sorafenib. SC-59 downregulated P-STAT3 and induced autophagy in all tested HCC cell lines. Furthermore, our in vivo data showed that both sorafenib and SC-59 inhibited tumor growth, downregulated P-STAT3, enhanced the activity of SHP-1 and induced autophagy in PLC5 tumors, suggesting that sorafenib and SC-59 activate autophagy in HCC. In conclusion, sorafenib and SC-59 induce autophagy in HCC through a SHP-1-STAT3-Mcl-1-Beclin 1 pathway.

Cell Death and Disease (2013) 4, e485; doi:10.1038/cddis.2013.18; published online 7 February 2013

Subject Category: Cancer

Hepatocellular carcinoma (HCC) is the fifth most common cancer worldwide and the third leading cause of cancer death. ${ }^{1}$ Incidence of HCC is strongly correlated with cirrhosis that results from causes such as chronic hepatitis $B$ virus $(\mathrm{HBV})^{2,3}$ and/or chronic hepatitis C virus (HCV) infection, alfatoxin exposure, alcoholic cirrhosis and cigarette smoking. ${ }^{1,4,5}$ As $\mathrm{HCC}$ is highly resistant to chemotherapy, targeted therapies have been evaluated as first-line treatments or combinational therapies. ${ }^{6-8}$ Sorafenib (Nexavar), a multiple kinase inhibitor, was approved by the US Food and Drug Administration for the treatment of advanced HCC in 2007, and is the first clinically approved targeted drug therapy for HCC. ${ }^{9,10}$ However, the precise mechanism by which sorafenib induces tumor cell death is still under investigation. We identified STAT3 as a major kinase-independent target of sorafenib through increasing $\mathrm{SH} 2$-containinig protein tyrosine phosphatase (SHP-1) activity. ${ }^{11,12}$ SHP-1 is a key negative regulator of STAT3 that can dephosphorylate p-STAT3 and further inhibit its downstream gene expression. ${ }^{13}$ We validated the role of the SHP-1/STAT3-related signaling pathway in the sorafenib-induced anti-HCC effect by several novel kinase-independent derivatives of sorafenib. ${ }^{14,15}$ These derivatives, which had no inhibitory effect on kinases such as the Raf and VEGFR families showed a similar or more potent antitumor effect than sorafenib through the activation of SHP-1 phosphatase activity.

Autophagy is an important catabolic process for the degradation of cytoplasmic proteins via autolysosomal digestion. ${ }^{16,17}$ Autophagy is initiated by the formation of a membranous cistern called the isolation membrane (or phagophore) that contains damaged cell components. Next, a nascent membrane is further fused to form a doublemembrane vesicle (called an autophagosome). The process of mammalian autophagy is divided into six principal steps: initiation, nucleation, elongation, closure, maturation and degradation. ${ }^{16,18}$ In addition to degradation thorough lysosomal machinery, autophagy has been reported to induce programmed cell death called autophagic cell death (ACD, programmed cell death type II). ${ }^{19-21}$ Beclin 1 (Atg6), a Bcl-2-homology domain $3(\mathrm{BH} 3)$ protein, interacts with Vps34 (a class III PI3K), Vps15 (a myristoylated kinase) and UV irradiation resistance-associated tumor suppressor gene (UVRAG) to form a core complex to allow autophagosome nucleation, a vital step of autophagy. ${ }^{22}$ However, Bcl-2 and

\footnotetext{
${ }^{1}$ Department of Medical Research, National Taiwan University Hospital, Taipei, Taiwan; ${ }^{2}$ National Center of Excellence for Clinical Trial and Research, National Taiwan University Hospital, Taipei, Taiwan; ${ }^{3}$ Institute of Biopharmaceutical Sciences, National Yang-Ming University, Taipei, Taiwan; ${ }^{4}$ Division of Hematology and Oncology, Department of Medicine, Taipei Veterans General Hospital, Taipei, Taiwan; ${ }^{5}$ School of Medicine, National Yang-Ming University, Taipei, Taiwan; ${ }^{6}$ School of Veterinary Medicine, National Taiwan University, Taipei, Taiwan and ${ }^{7}$ Department of Oncology, National Taiwan University Hospital, Taipei, Taiwan

*Corresponding author: K-F Chen, Department of Medical Research, National Taiwan University Hospital, No 7, Chung-Shan South Road, Taipei 100, Taiwan. Tel: +886 2 23123456, ext: 63548; Fax: +886 2 23225329; E-mail: kfchen1970@ntu.edu.tw

${ }^{8}$ These authors contributed equally to this work.

Keywords: SC-59; sorafenib; STAT3; HCC

Abbreviations: HCC, hepatocellular carcinoma; TRAIL, tumor necrosis factor-related apoptosis-inducing ligand; STAT3, signal transducers and activators of transcription 3; DMEM, Dulbecco's modified Eagle's medium; FBS, fetal bovine serum

Received 03.9.12; revised 05.1.13; accepted 09.1.13; Edited by A Rufini
} 
Bcl-xL can interact with Beclin 1 via the $\mathrm{BH} 3$ domain and inhibit the Beclin 1-containing core complex. In addition, the expression level of myeloid cell leukemia-1 (Mcl-1) has been suggested to regulate autophagic flux. Specifically, deletion of $\mathrm{Mcl}-1$ in cortical neurons of transgenic mice has been found to activate a robust autophagic response. ${ }^{23}$ The inhibition of $\mathrm{Mcl}-1$ is hypothesized to induce autophagic cell death.

In this study, we unraveled the molecular mechanism by which sorafenib induces autophagy in HCC cells. We found that sorafenib-induced degradation of $\mathrm{Mcl}-1$ disrupts its association with Beclin 1 and promotes significant autophagic cell death. Using a kinase-independent derivative of sorafenib, SC-59, we confirmed that this autophagic effect is related to the SHP-1/STAT3 signaling pathway. Both SC-59 and sorafenib resulted in disassociation of the $\mathrm{Mcl}-1-$ Beclin 1 complex and induced autophagic cell death in vitro and in vivo via a SHP-1/STAT3-dependent mechanism.

\section{Results}

Sorafenib induces autophagy in HCC cell lines. Autophagy is known to be able to either suppress or promote cancer cell growth depending upon cell status. First, to evaluate the potential autophagic effect of sorafenib in HCC cells, we measured the expression levels of LC3-I and LC3-II. In the four HCC cell lines tested, we found significant induction of LC3-II with sorafenib at a clinically relevant dose indicating that sorafenib increases autophagosome formation in HCC cell lines (Figure 1a). However, the expression level of Atg5, an essential factor for autophagosome formation, was not affected by sorafenib. Furthermore, sorafenib induced the formation of LC3-II in a time-dependent manner (Figure 1b, Left). Notably, the level of p62, a major selective substrate for autophagy that is incorporated into autophagosomes through direct binding to LC3, was decreased with sorafenib treatment. The p62 inhibition was inversely correlated with higher autophagic activity. The expression level of Beclin 1 and Atg5 were increased slightly with longer durations of sorafenib treatment (Figure 1b, left). To analyze the effect of sorafenib on autophagic flux, we further co-treated PLC5 cells with sorafenib and chloroquine (CQ). $\mathrm{CQ}$ is an autophagy inhibitor that blocks lysosomeautophagosome fusion and subsequent lysosomal protein degradation by raising lysosomal $\mathrm{pH}$ level. Sorafenib inhibited $C Q$-induced p62 and increased the level of the membrane-bound form of LC3 (LC3-II) compared to CQ alone (Figure $1 \mathrm{~b}$, middle). In addition to $\mathrm{CQ}$, we used another inhibitor of autophagy, bafilomycin $A 1$, to validate the autophagic effect of sorafenib. Combination of sorafenib and $A 1$ induced more LC3-II production than A1 alone in PLC5 and SK-Hep1 (Figure 1b, right). Most importantly, both $A 1$ and $C Q$ significantly reduced the effect of sorafenib on cell viability. Moreover, we monitored the number of autophagosomes formed on exposure to sorafenib. Using electron microscopy, apparent autophagic vacuoles that indicate autophagosomes and late-stage autolysosomes were observed in sorafenib-treated PLC5 cells (Figure 1c). The revealed autophagosomes contained undigested cytoplasmic components such as mitochondria and fragments of endoplasmic reticulum. As autophagy is characterized by the formation of acidic vesicular organelles (AVOs), we then stained PLC5 cells with acridine orange $(A O)$ to measure sorafenib-induced autophagy. Protonated AO becomes trapped on the low $\mathrm{pH}$ side of the membrane barrier leading to its accumulation in acidic organelle structures. As shown in Figure1d, protonated $A O$ dye fluoresced bright red in sorafenib-treated PLC5 cells. In contrast, no distinct AO-R was observed in non-treated cells. Together, these results confirmed that sorafenib induced autophagy in HCC cell lines.

\section{Sorafenib disrupts the Beclin 1-Mcl-1 complex through} inhibition of the p-STAT3-related signaling pathway. To elucidate the molecular mechanism by which sorafenib induces autophagy in HCC cell lines, we next assayed potential targets of sorafenib involved in the regulation of this autophagic effect. Previously, RAF/MEK/ERK-mediated signaling was implicated in the sorafenib-induced anticancer effect. ${ }^{24}$ Recently, other signaling pathways such as STAT3Mcl-1 have also been reported to be involved in the effect sorafenib. ${ }^{11,12,25,26}$ As shown in Figure2a, sorafenib inhibited p-STAT3-Mcl-1 in a dose-dependent manner. It is also possible that sorafenib affects other apoptosis-related molecules in HCC cell lines. We found that the p-Akt/mTOR-related signaling pathway did not participate in the sorafenib-induced molecular events (Figure $2 \mathrm{~b}$ ). The expression statuses of p-Akt (T308)/Akt, p-mTOR (S2481)/ mTOR, p-S6/S6, 4EBP1 and TSC1 were not affected by sorafenib treatment. As expected, sorafenib inactivated ERK at a higher dose $(10 \mu \mathrm{M})$. Beclin 1 (the mammalian ortholog of yeast Atg6), a Bcl-2-homology domain 3 (BH3)-only protein, is a key factor in the autophagy process. Beclin 1containing complex (also called PI3K complex III), which contains Vps34, Vps15, UVRAG and inhibitory Bcl-2/Bcl-XL, contributes to vesicle nucleation in the initial step of autophagy. ${ }^{18,27}$ Recently, Mcl-1, an anti-apoptotic Bcl-2 homolog, has been reported to have a vital role in the regulation of autophagy. The degradation of $\mathrm{Mcl}-1$ relieves Beclin 1 and thus promotes the formation of the nucleated core complex. ${ }^{23}$ To determine whether sorafenib induces autophagy via this mechanism, we investigated the association between $\mathrm{Mcl}-1$ and Beclin 1. As shown in Figure 2c, sorafenib significantly disrupts the interactions between $\mathrm{Mcl}-$ 1 and Beclin 1. Treatment of sorafenib at $20 \mu \mathrm{M}$ for 16 or $48 \mathrm{~h}$ diminished the interactions between Mcl-1 and Beclin 1 (Figure 2c, middle upper). Considering that high-dose sorafenib significantly affected the expression level of Mcl1 , we also examined the association status between Beclin 1 and $\mathrm{Mcl}-1$ with lower concentration of sorafenib $(10 \mu \mathrm{M})$. The decreased level of $\mathrm{Mcl}-1$ in IP-Beclin 1-containing complex was also found in sorafenib-treated PLC5 cells (Figure 2c, middle lower). In addition, we also analyzed the protein-protein interactions between Beclin 1 and $\mathrm{Mcl}-1$ by immunoprecipitating $\mathrm{Mcl}-1$. We found that sorafenib reduced the interactions between Beclin 1 and $\mathrm{Mcl}-1$. (Figure 2c, right). These data imply that sorafenib inhibits the expression of $\mathrm{Mcl}-1$ via its transcription factor, STAT3, thereby relieving inhibition of Beclin 1 and promoting further formation of autophagosomes. Notably, Beclin 1 and its other inhibitors such as Bcl-XL were not affected by 
a
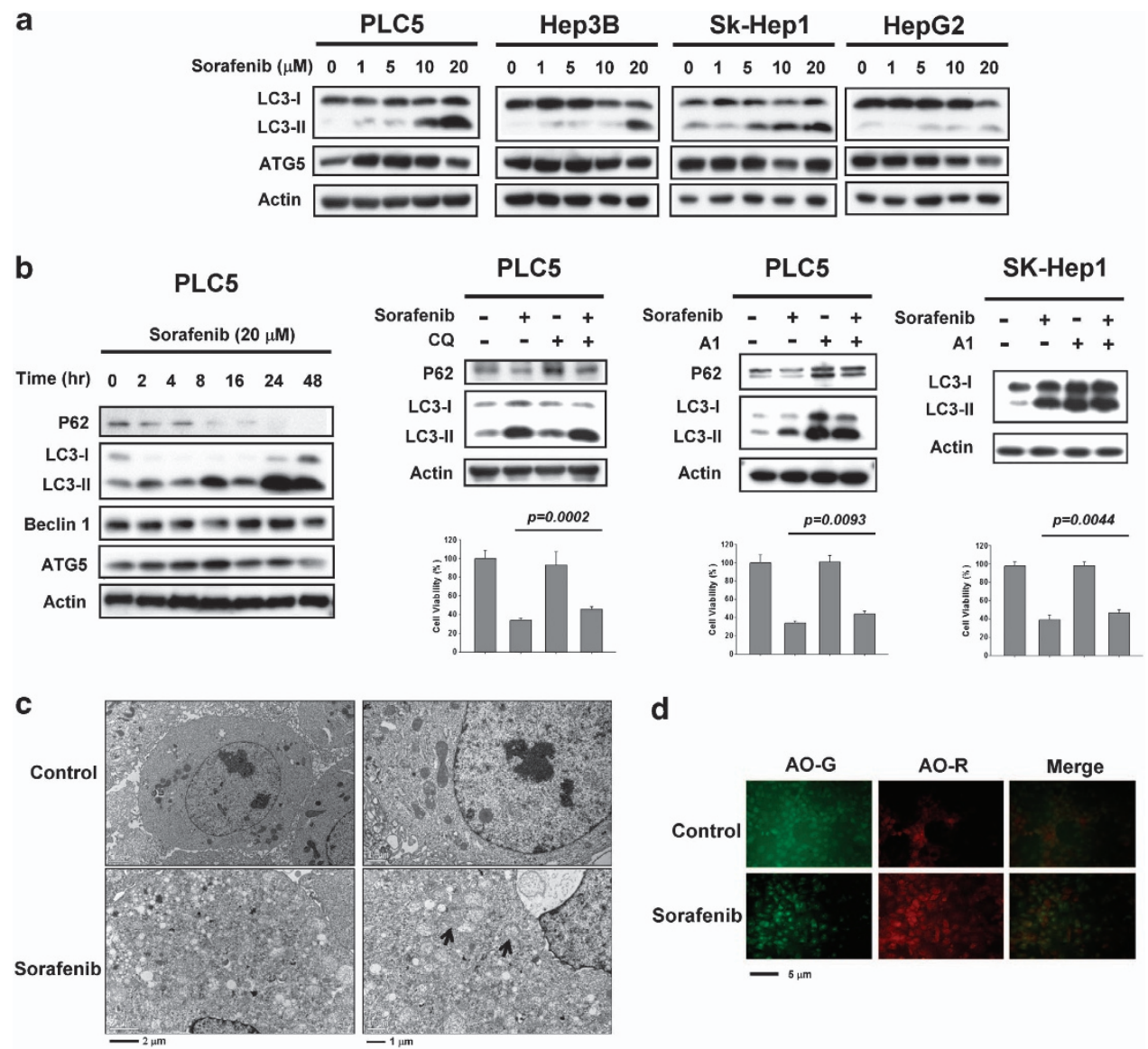

d

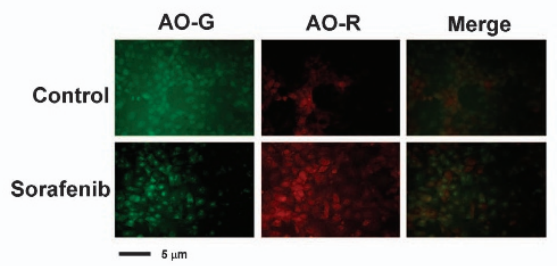

Figure 1 Sorafenib induces autophagy in HCC cells. (a) Sorafenib induces the conversion of LC3 in a dose-dependent manner. PLC5, Hep3B, SK-Hep1 and HepG2 were exposed to sorafenib at the indicated doses for $16 \mathrm{~h}$ and the expression levels of LC3-II were analyzed by western blot. (b) Time-dependent effect of sorafenib on autophagyrelated proteins. PLC5 cells were exposed to $20 \mu \mathrm{M}$ sorafenib for various periods of time (left). Sorafenib activates autophagic flux in PLC5 cells (middle). Western blot showing P62 degradation and LC3 lipidation in PLC5 cells treated with sorafenib and/or lysosomal inhibitor. The cells were treated with or without $20 \mu \mathrm{M}$ sorafenib in the presence or absence of $10 \mu \mathrm{M}$ chloroquine (CQ) for $16 \mathrm{~h}$. PLC5 or SK-Hep1 cells were treated with or without $20 \mu \mathrm{M}$ sorafenib in the presence or absence of $20 \mathrm{nM}$ bafilomycin A1 (A1) for $16 \mathrm{~h}$ (right). The effect of CQ or A1 on cell viability was performed in MTT assay. (c) Transmission electron microscopy (TEM) images showing autophagosome (arrow) formation in PLC5 cells treated with $20 \mu \mathrm{M}$ sorafenib for $16 \mathrm{~h}$. (d) Images of acridine orange (AO) staining of HCC cells after $16 \mathrm{~h}$ treatment with $20 \mu \mathrm{M}$ sorafenib as detected by fluorescence microscopy. AO-R indicates acidic vesicular organelle formation

sorafenib (Figure 2c, left). These results imply that sorafenib induces STAT3-dependent inhibition of $\mathrm{Mcl}-1$, hence relieving its association with Beclin 1 to activate autophagy in HCC cell lines.

SHP-1-dependent inhibition of STAT3 mediates autophagic cell death induced by sorafenib. To further clarify the molecular mechanism by which sorafenib induces autophagy in HCC cell lines, we next investigated whether the SHP-1/STAT3 signaling pathway has a part in sorafenibinduced autophagy. First, we assessed the effect of inhibition of p-STAT3 on autophagy. Both sorafenib and STAT3 inhibitor III, WP1066, treatment resulted in significant conversion from LC3-I to LC-II (Figure 3a, top). Notably, this specific STAT3 inhibitor (WP1066) induced an evident amount of LC-II suggesting that inhibition of STAT3 signaling prompts autophagy in HCC cells. On the other hand, PLC5 cells with ectopic expression of STAT3 were insensitive to sorafenib-induced autophagy (Figure 3a, bottom). Together, our results suggest a potential interplay whereby sorafenib induces an autophagic effect via inactivation of STAT3. It is important to note that sorafenib inhibits the STAT3-related signaling pathway through increasing SHP-1 phosphatase activity, $^{12,14}$ meaning that activated SHP-1 may also be involved in sorafenib-induced autophagy. As demonstrated in Figure 3b, silencing SHP-1 with specific siRNA significantly restored the expression level of LC-II under sorafenib treatment. These data indicate that the SHP-1/STAT3-related pathway also has a vital role in sorafenib-induced autophagy.

The results shown in Figure $2 \mathrm{c}$ confirmed that sorafenib disrupts the interaction between Mcl-1 and Beclin 1 and suggest that relieving Beclin 1 is involved in sorafenib-induced autophagy. To further validate the role of $\mathrm{Mcl}-1$ and Beclin 1 in sorafenib-induced autophagy, we assayed overexpression of Mcl-1 and knockdown of Beclin 1, respectively. Importantly, the expression level of LC-II was almost completely abolished in PLC5 cells expressing ectopic Mcl-1 (Figure 3c). Sorafenib can not induce potent autophagy in the presence of $\mathrm{Mcl}-1$. Additionally, silencing Beclin 1 in HCC cells also inhibited sorafenib-induced autophagy (Figure 3d, upper). Notably, 


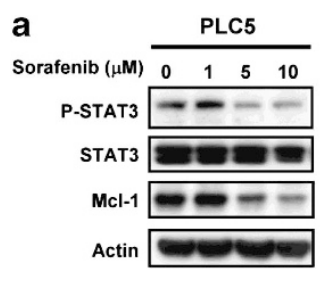

c

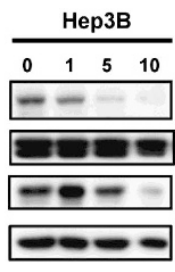

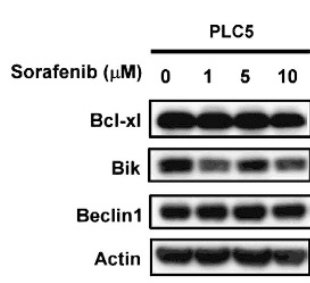
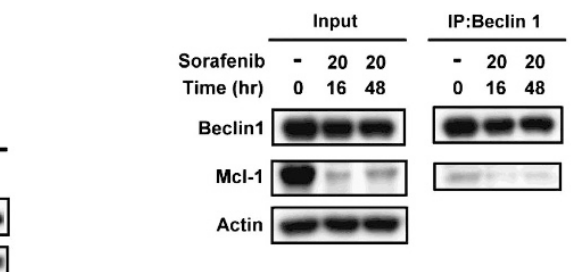

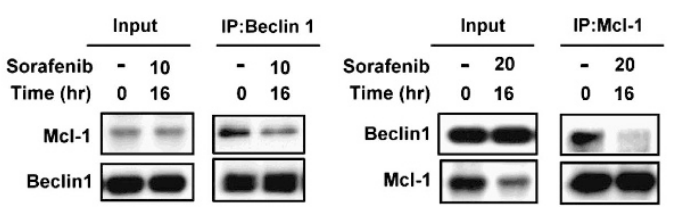

b

\begin{tabular}{lllll}
\cline { 2 - 4 } Sorafenib $(\mu \mathrm{M})$ & 0 & 1 & 5 & 10
\end{tabular}

P-ERK $\approx=0$

ERK2 $=-\infty-$

P-Akt(T308) $\square$

P-Akt(S473)

Akt1

TSC1 $=\mathrm{met}$

P-TSC2 $--2-$

TSC2

P-mTOR(S2481) - - -

mTOR

P-S6

s6

4EBP1 $-\cdots-\infty$

Actin

Figure 2 Sorafenib disrupts the Beclin 1-Mcl-1-complex through inhibition of the p-STAT3-related signaling pathway. (a) Effects of sorafenib on STAT3-related proteins in HCC cells. The cells were treated with sorafenib at the indicated dose for $16 \mathrm{~h}$. (b) Effects of sorafenib on the Akt/mTOR-related signaling pathway in PLC5 cells. The cells were treated with sorafenib at the indicated dose for $16 \mathrm{~h}$. (c) Effects of sorafenib on Bcl-2 related proteins (left). The cells were treated with sorafenib at the indicated dose for $16 \mathrm{~h}$. Sorafenib does not affect the amount of Bcl-xl, Bik and Beclin 1. Sorafenib induces the disassociation of Beclin 1 and Mcl-1 (middle). Beclin 1 was immunoprecipitated from PLC5 cells treated with 10 or $20 \mu \mathrm{M}$ sorafenib at $16 \mathrm{~h}$ or $48 \mathrm{~h}$ and analyzed for the presence of Mcl-1 and Beclin 1 . Mcl-1 was immunoprecipitated from PLC5 cells treated with $20 \mu \mathrm{M}$ sorafenib at $16 \mathrm{~h}$ and analyzed for the presence of Mcl-1 and Beclin 1 (right)

silencing of Beclin 1 reversed sorafenib-induced cell toxicity as evident by MTT assay (Figure 3d, lower). There was decreasing conversion of LC3-I to LC3-II in the absence of Beclin 1, which indicates that free-form Beclin 1 is a determinant of sorafenib-induced autophagy. Together these results confirm that SHP-1/STAT3-dependent signaling is involved in sorafenib-induced autophagy, suggesting that STAT3-driven $\mathrm{Mcl}-1$ was also inhibited, resulting in the release of Beclin 1, allowing Beclin 1 to form a core complex with other interaction proteins for autophagosome formation.

SC-59, a kinase-independent derivative of sorafenib, induces more autophagic cell death than sorafenib. Recently, we applied the kinase-independent mechanism of SC-1 as a molecular basis from which to develop a novel class of SHP-1 activators. ${ }^{14,15}$ The replacement of N-methylpicolinamide with a phenylcyano group abolished kinase activity while retaining phospho-STAT3 repressive activity. Screening of these derivatives revealed that SC-59 in particular had a potent autophagic effect in HCC cell lines. To further address the effect of SC-59 on autophagic cell death, here we performed more specific assays to validate the molecular mechanism of SC-59. The difference in chemical structure between sorafenib and SC-59 is shown in Figure $4 \mathrm{a}$. The kinase-independent characteristic of SC-59 was confirmed by Raf-1 activity (Figure $4 a$, right). In four HCC cell lines, SC-59 showed more significant cytotoxicity than sorafenib in a dose-escalation manner (Figure 4b). In addition, SC-59 exhibited better effects than sorafenib on apoptosis in a dose-dependent manner (Figure 4c). SC-59 demonstrated substantial apoptosis in a dose-dependent manner. SC-59 also induced the inhibition of p-STAT3 and potential autophagy as a result of more conversion to LC3-II (Figure $4 \mathrm{~d}$, upper). At the same dose $(10 \mu \mathrm{M})$, SC-59 displayed a more potent effect on autophagy than sorafenib in all four HCC cell lines (Figure 4d, lower). We also found distinct evidence of autophagosome formation using electron microscopy (Figure 4e, upper) and AO staining (Figure 4e, lower) after SC-59 treatment. We found that SC-59 also induced autophagy with co-treatment of CQ. Importantly, SC-59-induced cell viability change was reversed by adding $\mathrm{CQ}$ (Figure 4f, left). Further, A1 also rescued the effect of SC-59 on cell toxicity in PLC5 and SK-Hep1 (Figure 4f, right). Therefore, we propose that the anti-HCC effect of SC-59 is correlated with autophagic cell death.

Relieving Beclin 1 results in SC-59-induced autophagy via a SHP-1/STAT3/Mcl-1-dependent signaling pathway. To investigate the molecular mechanism by which SC-59 induces an anti-HCC effect, we assayed the impact of SHP-1/STAT3-dependent signaling on SC-59-induced autophagy. First, we investigated whether inactivation of STAT3 was related to SC-59-induced autophagy. Both SC59 and WP1066 showed the conversion from LC3-I to LC3-II (Figure 5a, left). In contrast, SC-59 did not induce evident LC3-II in PLC5 cells ectopically expressing STAT3 (Figure 5a, left). Meanwhile, SC-59 lost its autophagic effect in the absence of SHP-1. We did not find distinct expression of LC3-II in PLC5 cells with silenced SHP-1 (Figure 5a, right). As activation of SHP-1 has been found to be part of a major kinase-independent mechanism of action of this sorafenib derivative, we further assayed the effect of SC-59 on SHP-1 phosphatase activity. As expected, we observed that SC-59 increased SHP-1 phosphatase activity in a dose-dependent 
a

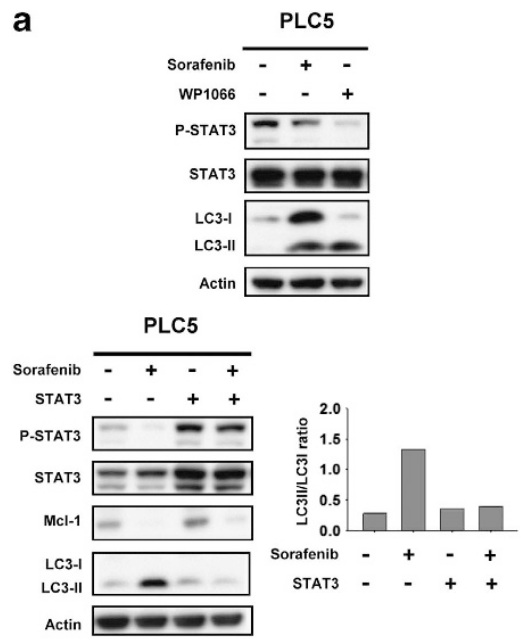

C

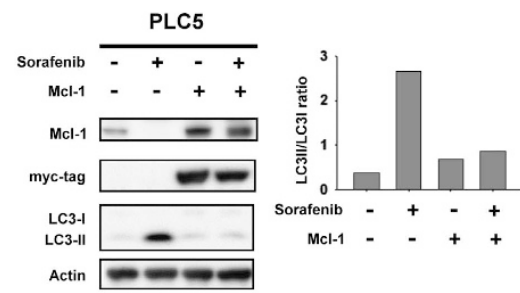

b

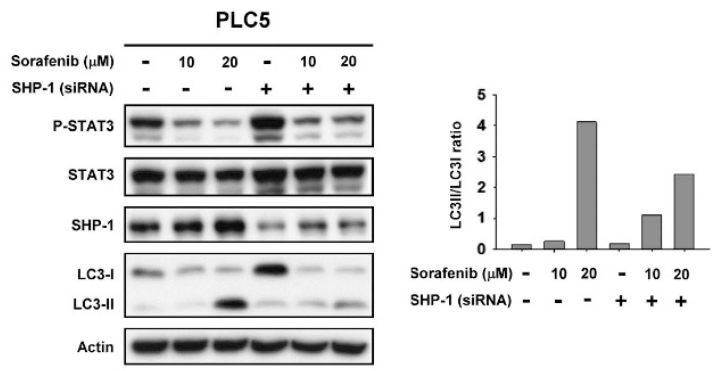

d

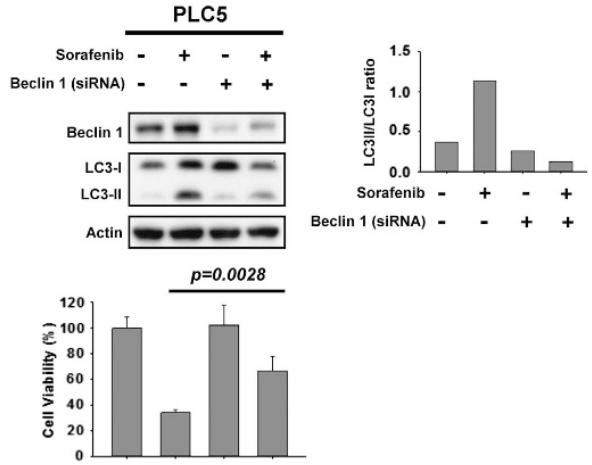

Figure 3 Inhibition of $p$-STAT3 is involved in sorafenib-induced autophagy through the induction of SHP phosphatase activity. (a) Specific inhibition of $p$-STAT3 induces significant autophagic activity. WP1066, a specific inhibitor of STAT3, induces autophagy in PLC5 cells (top). Cells ectopically expressing STAT3 were treated with sorafenib at $10 \mu \mathrm{M}$ for $16 \mathrm{~h}$ and the level of expression of LC3-II was measured (bottom). (b) Silencing SHP-1 by siRNA reduces the effect of sorafenib on p-STAT3 and LC3-II in HCC cells. PLC5 cells were transfected with control siRNA or SHP-1 siRNA for $24 \mathrm{~h}$ and then treated with sorafenib for $16 \mathrm{~h}$. (c) Effects of Mcl-1 on autophagy induced by sorafenib in HCC cells. Wild-type PLC5 or PLC5 transiently transfected with Mcl-1 were treated with sorafenib at $10 \mu \mathrm{M}$ for $16 \mathrm{~h}$. (d) Silencing Beclin 1 by siRNA reduces the effects of sorafenib on LC3-II in HCC cells. PLC5 cells were transfected with control siRNA or Beclin 1 siRNA for $24 \mathrm{~h}$, then treated with sorafenib at $10 \mu \mathrm{M}$ for $16 \mathrm{~h}$. The effect of siBeclin 1 on cell viability was demonstrated in MTT assay

manner in both PLC5 cells and SHP-1-containing IP complex (Figure 5b). Notably, SC-59 induced more potent phosphatase activity than sorafenib incubation in vitro.

As sorafenib disrupted the interaction between Beclin 1 and Mcl-1, we further investigated whether SC-59 also affects this association to induce autophagy. As shown in Figure $5 \mathrm{c}$, SC-59 treatment decreased the level of Mcl-1 in Beclin 1-containing complex, suggesting that SC-59 releases more free-form Beclin 1 through Mcl-1 inhibition and thus promotes autophagy. To further confirm the roles of Mcl-1 and Beclin 1 in this autophagic effect, we transfected either ectopic $\mathrm{Mcl}-1$ or siBeclin 1 into PLC5 cells to observe the effect of SC-59 on autophagy. Both overexpression of $\mathrm{Mcl}-1$ and silencing Beclin 1 in HCC cells almost fully restored the conversion from LC3-I to LC3-II induced by SC-59, indicating that the inhibitory effect of $\mathrm{Mcl}-1$ is a key basis for autophagy induced by sorafenib and its derivatives (Figure $5 \mathrm{~d}$, Upper).

The knockdown of Beclin 1 also significantly reversed the effect of SC-59 on cell survival in PLC5 cells (Figure 5d, lower). To examine the effect of sorafenib and SC-59 on $\mathrm{Mcl}-1$, we investigated the effect of sorafenib or SC-59 on the transcription of $\mathrm{Mcl}-1$. Our data showed that sorafenib or
SC-59 significantly decreased mRNA levels of Mcl-1 in a timedependent manner (Figure $5 \mathrm{e}$, right). Notably, the treatment of sorafenib or SC-59 did not alter the degradation of Mcl-1 significantly (Figure $5 \mathrm{e}$, left).

Taken together, we propose that sorafenib and its derivative, SC-59, inhibit the expression of Mcl-1 and further release Beclin 1 to form a nucleated core complex through a SHP-1/STAT3-dependent signaling pathway. Moreover, based on the premise that SC-59 acts in a kinase-independent manner, we propose a specific role for SHP-1/STAT3 in autophagic cell death that accounts for the observation of more cytotoxicity and LC-II in SC-59 than sorafenib-treated cells.

Sorafenib and SC-59 induce significant tumor growth inhibition via SHP-1-dependent autophagic cell death. To verify tumor growth inhibition by sorafenib and its derivative SC-59, we applied these two drugs to HCC-bearing mice and evaluated the biological effect in vivo. SC-59 showed more potent tumor growth inhibition than sorafenib at the same dose $(20 \mathrm{mg} / \mathrm{kg} /$ day) (Figure $6 \mathrm{a}$, left). Autophagic vesicles were observed in tumors treated 
a

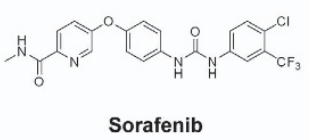

b

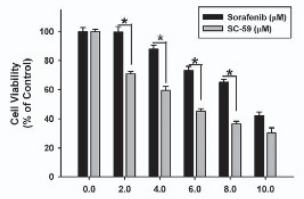

C

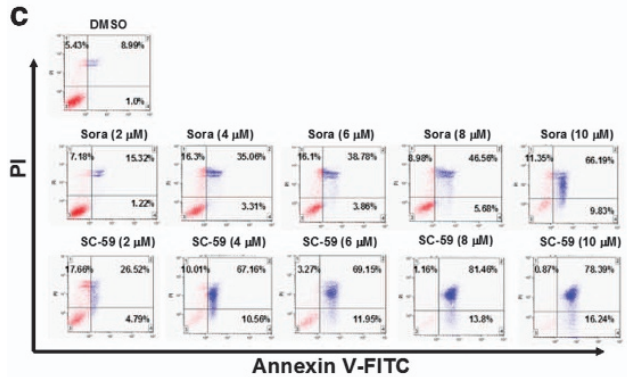

Annexin V-FITC
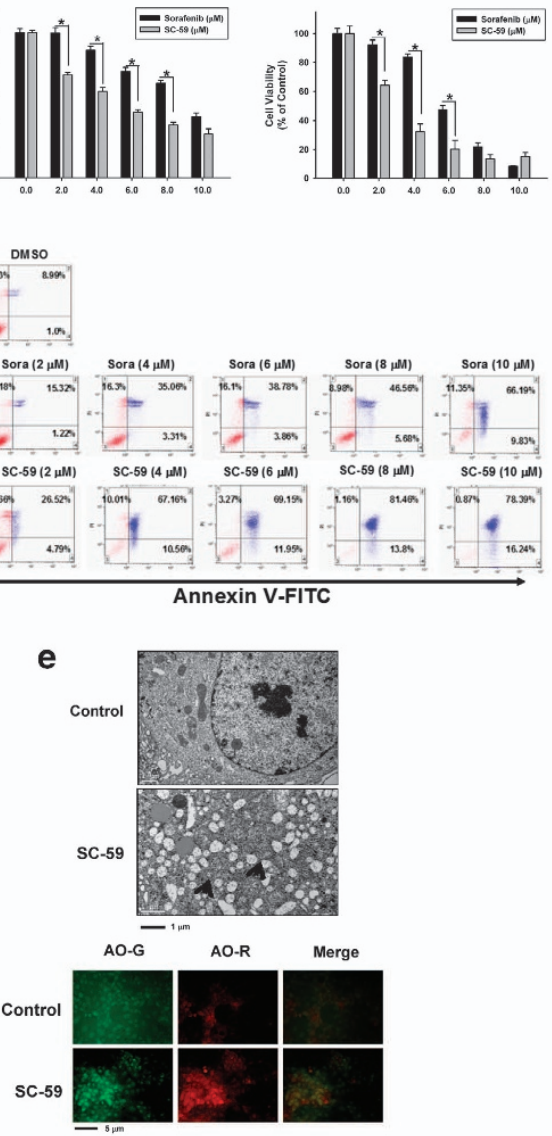

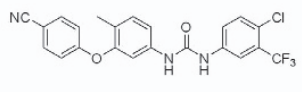

SC-59

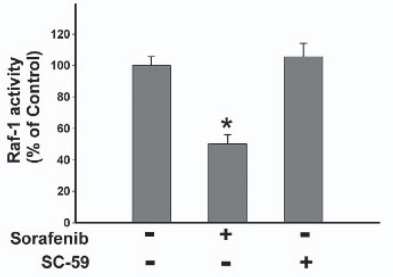

HepG2

Hep3B
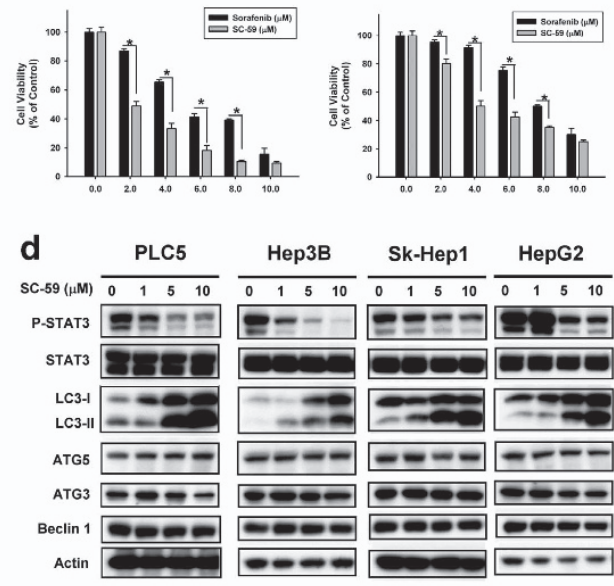

PLC5 Sk-Hep1 HepG2 Hep3B
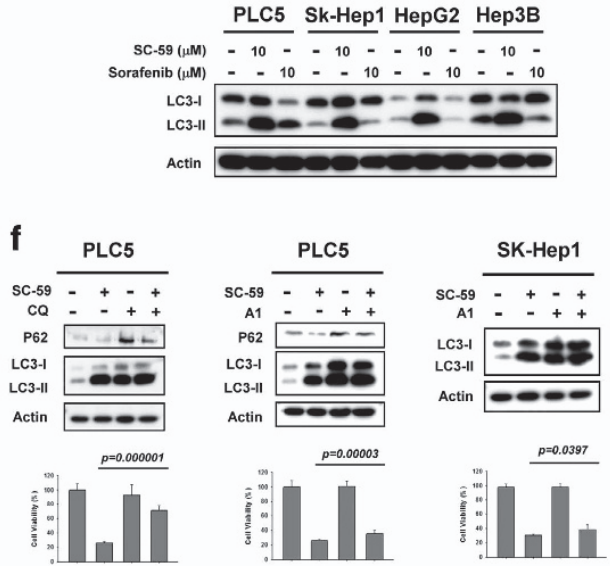

Figure 4 SC-59, a kinase-independent derivative of sorafenib, is a more effective anticancer agent than sorafenib in HCC cells. (a) Chemical structures of sorafenib and SC-59 (left). Effect of sorafenib and SC-59 on Raf-1 activity. Hep3B cells were treated with sorafenib or SC-59 at $10 \mu \mathrm{M}$ for $24 \mathrm{~h}$ (right). Columns, mean; bars, S.D. ( $n=3$ ). ${ }^{*} P<0.05$. (b) The cell viability of sorafenib and SC-59 in four HCC cell lines. The cells were treated with sorafenib or SC-59 at indicated dose for $48 \mathrm{~h}$ and cell viability was assessed by MTT assay. Columns, mean; bars, S.D. $(n=8)$. ${ }^{*} P<0.05$. (c) Both sorafenib and SC-59 induced significant apoptosis in a dose-dependent manner. The early and late apoptosis was observed in sorafenib or SC-59-treated PLC5 cells. (d) SC-59 downregulates p-STAT3 and increases LC3-II in HCC cells (upper). The cells were treated with SC-59 at indicated dose for $16 \mathrm{~h}$. SC-59 induces more LC3-II than sorafenib. The cells were treated with $10 \mu \mathrm{M} \mathrm{SC}-59$ or sorafenib for $16 \mathrm{~h}$ (lower). (e) Transmission electron microscopy images showing autophagosome (arrow) formation in PLC5 cells treated with $10 \mu \mathrm{M} \mathrm{SC}-59$ for $16 \mathrm{~h}$ (upper). fluorescence microscopy images of $\mathrm{AO}$ staining in $\mathrm{HCC}$ cells after $16 \mathrm{~h}$ treatment with $10 \mu \mathrm{M} \mathrm{SC}-59$ (lower). AO-R indicates acidic vesicular organelle formation, which demonstrates the formation of autolysosomes. (f) SC-59 activates autophagic flux in PLC5 and SK-Hep1 (left). Western blot showing P62 degradation and LC3 lipidation in PLC5 cells treated with SC-59 and/or lysosomal inhibitor. The cells were treated with or without $10 \mu \mathrm{M} \mathrm{SC}-59$ in the presence or absence of $10 \mu \mathrm{M}$ chloroquine (CQ) for $16 \mathrm{~h}$. PLC5 or SK-Hep1 cells were treated with or without $10 \mu \mathrm{M} \mathrm{SC}-59$ in the presence or absence of $20 \mathrm{nM}$ Bafilomycin A1 (A1) for $16 \mathrm{~h}$ (right). The effect of CQ or A1 on cell viability was performed in MTT assay

with sorafenib and SC-59 by TEM (Figure 6a, right). These data indicate sorafenib and SC-59 induced significant autophagy in vivo. Importantly, we found significant inhibition of p-STAT3 and Mcl-1 in both the sorafenib and SC-59treated tumor samples (Figure $6 \mathrm{~b}$, left). The conversion from LC3-I to LC3-II was also demonstrated in both treatments. The kinase-independent derivative SC-59, showed a stronger effect on the SHP-1/STAT3-related signaling pathway, and displayed more potent autophagic cell death through an increased level of LC3-II (quantitative data in Figure $6 \mathrm{~b}$, middle). We also found more significant induction of SHP-1 activity in SC-59-treated tumor samples (Figure 6c). These data indicate that both sorafenib and SC-59 show an important anti-HCC effect in vivo; and the 
a

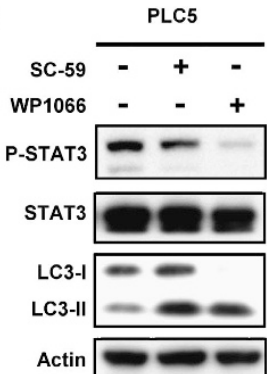

b

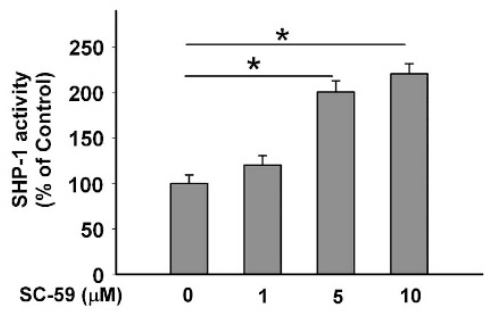

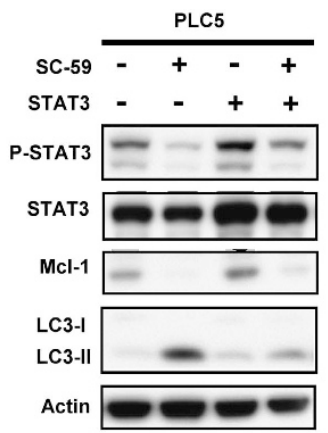

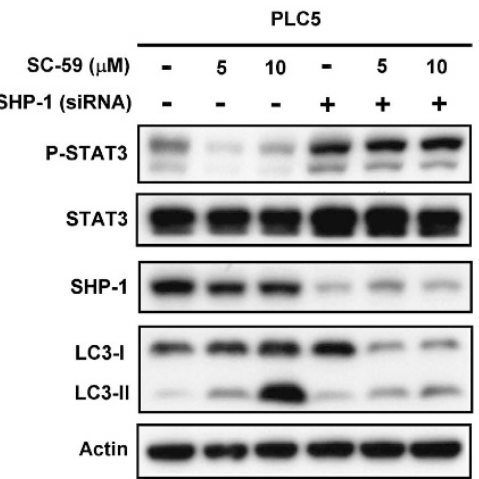

IP: SHP-1

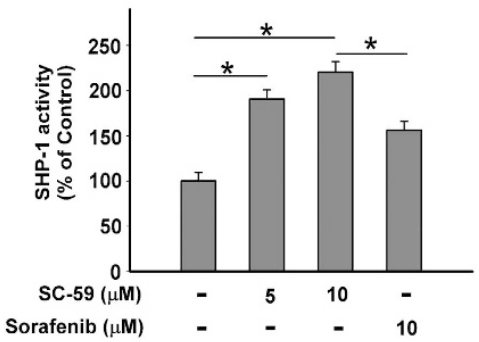

C

d

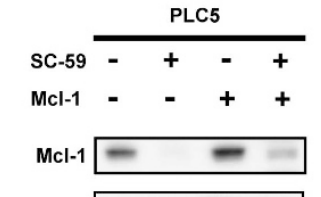

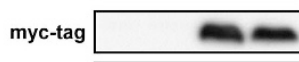

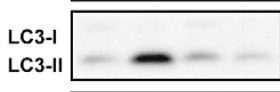

Actin

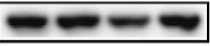

PLC5
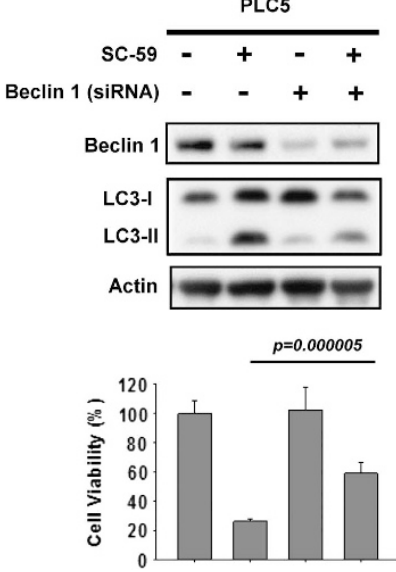

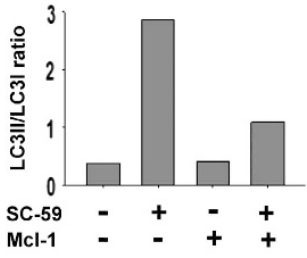

e

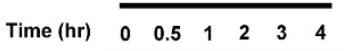

Mc

Actin
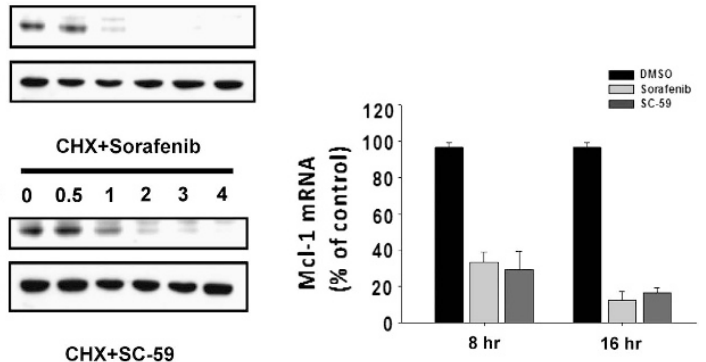

$\begin{array}{lllllll}\text { Time (hr) } & 0 & 0.5 & 1 & 2 & 3 & 4\end{array}$
$\mathrm{CHX}+\mathrm{SC}-59$

Mcl-1 - -

Actin

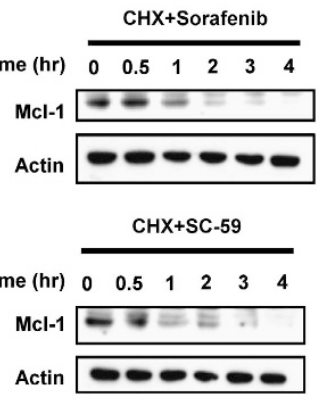

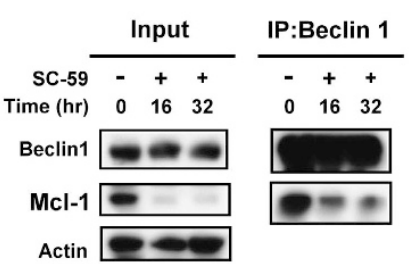


a

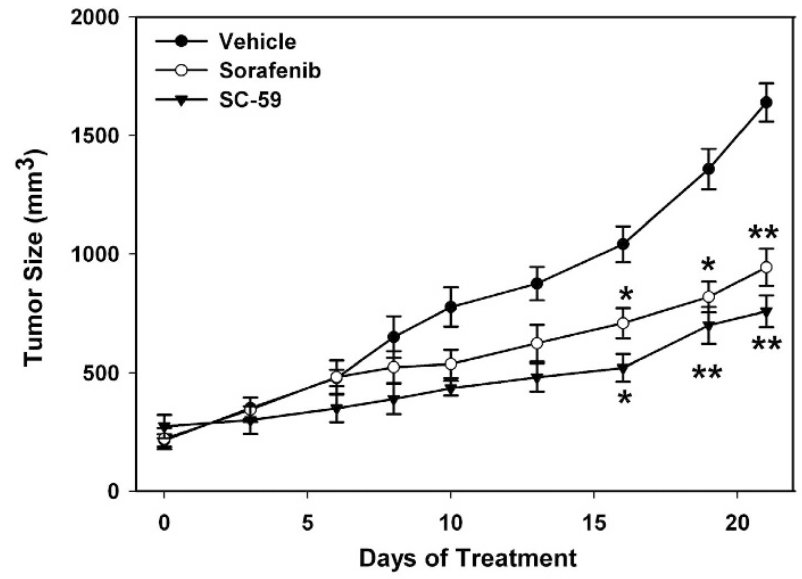

b
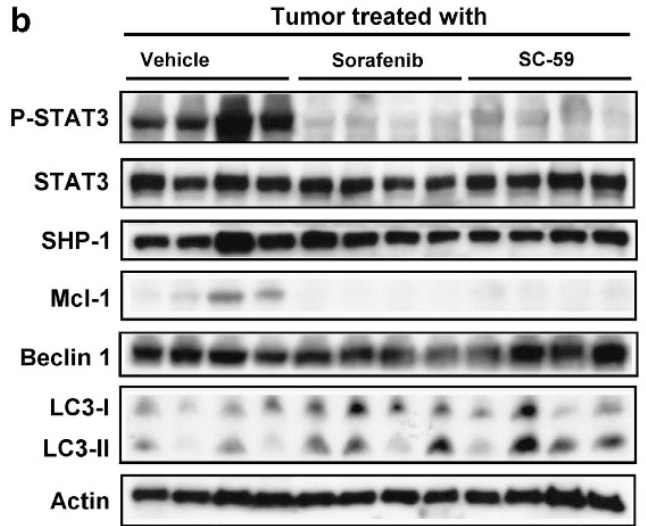
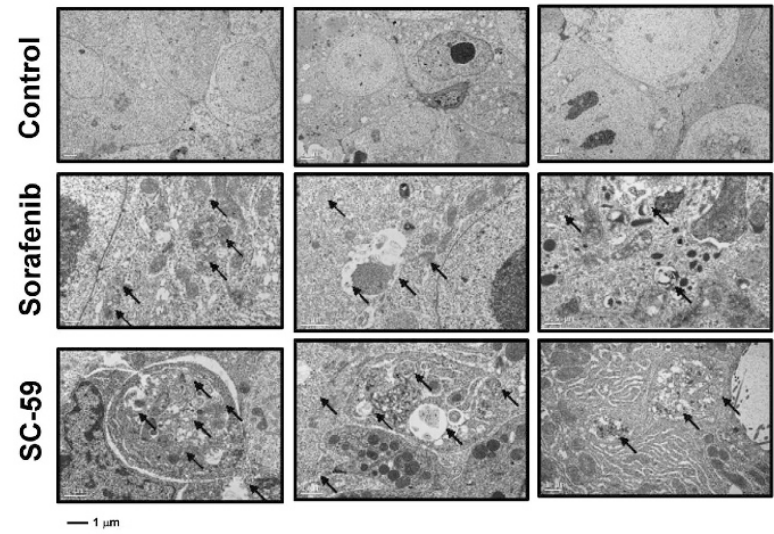

C
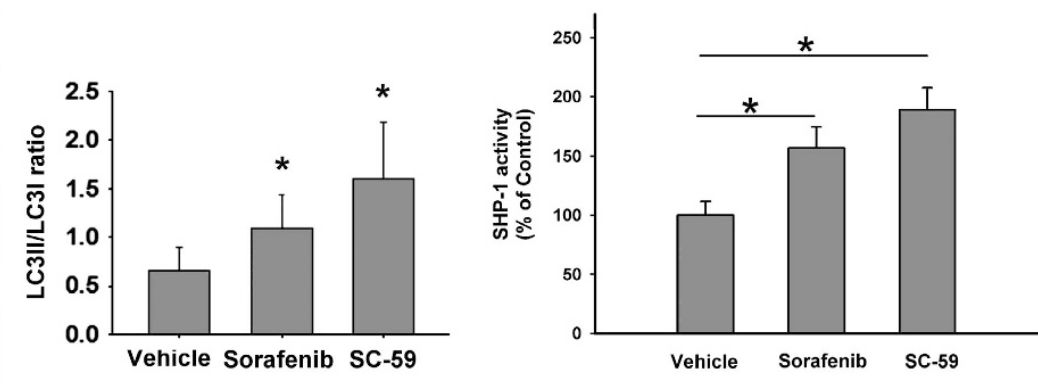

Figure 6 SC-59 exhibits more potent tumor growth inhibition and reveals significant SHP-1 activity and higher levels of expression of LC3-II than sorafenib in HCC-bearing mice. (a) Sorafenib $(20 \mathrm{mg} / \mathrm{kg})$ and SC-59 $(20 \mathrm{mg} / \mathrm{kg})$ inhibit PLC5 tumor growth (left). Points, mean; bars, S.E. $(n=10) .{ }^{*} P<0.05$. ${ }^{* \star} P<0.01$. SC-59 and sorafenib-induced autophagosomes were demonstrated in the tumor sample (right). (b) Western blot analysis of p-STAT3, STAT3, SHP-1, Mcl-1, Beclin 1 and LC3 in PLC5 tumors. (c) SHP-1 activity in PLC5 tumors. Columns, mean; bars, S.D. $(n=10) .{ }^{*} P<0.05$

critical role of SHP-1/STAT3-related signaling in autophagic cell death was also proved in this preclinical animal model.

\section{Discussion}

In this study, we proposed a molecular mechanism for the induction of autophagy by sorafenib. First, we validated the effect of sorafenib on autophagy by measuring: (1) the conversion of the cytoplasmic form of LC3 (LC3-I) to preautophagosomal/autophagosomal membrane-bound LC3 (LC3-II); (2) the autophagic degradation of p62; (3) electron microscopy of autophagosomes and (4) AO staining to monitor AVOs. Next, we further confirmed that sorafenib disrupts the interaction between Beclin 1 and $\mathrm{Mcl}-1$, suggesting that more relieved Beclin 1 is available to promote autophagosome formation. STAT3-dependent inhibition of $\mathrm{Mcl}-1$ caused the release of Beclin 1 from the Beclin 1-Mcl-1 complex as demonstrated in sorafenib-treated PLC5 cells (Figure 2c). Importantly, both overexpression of Mcl-1 and silencing of Beclin 1 almost completely abolished autophagy induced by sorafenib (Figures $3 c$ and d). Based on the results obtained with SC-59, a kinase-independent derivative of sorafenib, we further confirmed the molecular effect of SHP-1/STAT3-related signaling on sorafenib-induced autophagy. SC-59 had a more potent effect on SHP-1 phosphatase activity and higher levels of autophagy compared to sorafenib (Figure 4). Knockdown of SHP-1 in HCC cells inhibited the conversion of LC3-I to LC3-II, suggesting that activated SHP-1 has a vital role in sorafenib/SC-59induced autophagy. In the HCC xenograft, significant tumor growth inhibition was observed in mice treated with sorafenib or SC-59. The more potent SC-59 showed significant induction of autophagic marker, LC3-II, and SHP-1 phosphatase activity in vivo (Figure 6). These results suggest that the SHP-1/STAT3/Mcl-1 signaling pathway takes part in sorafenib-induced autophagic cell death via relieving of Beclin 1 both in vitro and in vivo.

Previously, sorafenib has been reported to induce both apoptosis and autophagy in human HCC cells via ERK1/2independent ER stress. ${ }^{28}$ This report suggests that sorafenibinduced autophagy alleviates ER stress-induced cell death and has a role in the molecular basis of resistance. Consequently, the combination of sorafenib with the autophagy inhibitor chloroquine (CQ) produced more pronounced tumor suppression in $\mathrm{HCC}$ both in vivo and in vitro. It has also been reported that autophagic activity triggered by sorafenib conferred a survival advantage to HCC cells. ${ }^{29}$ However, based on our results for the kinase-independent derivative of 
sorafenib, SC-59, we propose that activated autophagic flux is correlated with a potent apoptotic effect.

Beclin 1, carrying one $\mathrm{BH} 3$ domain, is a key initial protein involved in autophagy and the Beclin 1-containing core complex conducts the nucleation step in autophagosome formation. ${ }^{30,31}$ Importantly, anti-apoptotic proteins of the $\mathrm{Bcl}-2$ family such as Bcl-2 and $\mathrm{Bcl}-\mathrm{xL}$ can interact with Beclin 1 at residues 114-123 within the $\mathrm{BH} 3$ domain and inhibit the function of the Vps34--UVRAG-Beclin 1 nucleated core complex. ${ }^{22,27,32,33}$ Moreover, the phosphorylation of Beclin 1 at $\mathrm{T} 119$ within $\mathrm{BH} 3$ domain by DAPK was reported to dissociate Beclin 1 from inhibitory $\mathrm{Bcl}-\mathrm{xL}$ and promote autophagic activity. ${ }^{34}$ In addition, $\mathrm{Mcl}-1$ also has an inhibitory effect on the Beclin 1-associated core complex. For example, the expression of $\mathrm{Mcl}-1$ protected against the combination of lapatinib and obatoclax lethality. Lapatinib and obatoclaxinitiated autophagy was found to depend on NOXA-mediated displacement of the pro-survival BCL-2 family member, MCL-1, from beclin 1.35 In our data, we propose that degradation of $\mathrm{Mcl}-1$ is crucial for sorafenib-induced autophagy by releasing Beclin 1. This effect also determines autophagic cell death in the kinase-independent derivative, SC-59.

Previously, we proposed that the SHP-1/STAT3-related signaling pathway is a major target for inhibition of Mcl-1 and promotion of cell death in HCC. ${ }^{26}$ However, little is known about the interplay between STAT3 and autophagy. The inhibition of STAT3 and $\mathrm{mTOR}$ were reported to be involved in telomere 3'-overhang-specific DNA oligonucleotide (T-oligo)induced cancer cell death. ${ }^{36}$ In contrast, ROS-dependent activated STAT3 was also observed in cells treated with rapamycin or under starvation to promote the autophagic process. $^{37}$ We found that WP1066, a specific JAK/STAT3 inhibitor, as well as sorafenib can induce a significant expression level of LC3-II, and that, ectopic expression of STAT3 can further restore sorafenib-induced autophagy, which indicates that inhibition of p-STAT3 has a vital role in autophagic activity. In addition, SHP-1, a negative regulator of STAT3, is also implicated in the promotion of sorafenibinduced autophagy. Silencing SHP-1 almost fully abolished the conversion of LC3 induced by sorafenib (Figure 3b). Moreover, the higher levels of autophagy induced by SC-59 were correlated to anti-HCC effect in vitro and in vivo.

In this study, we proposed a molecular mechanism for the induction of autophagic cell death by sorafenib in HCC. Both sorafenib and its derivative induced the inhibition of $\mathrm{Mcl}-1$ via a SHP-1/STAT3-related pathway and released Beclin 1 to promote autophagosome formation. This study thus suggests that the disassociation of $\mathrm{Mcl}-1$ and Beclin 1 manages sorafenib-induced autophagy in HCC.

\section{Materials and Methods}

Reagents and antibodies. Sorafenib (Nexavar) was kindly provided by Bayer Pharmaceuticals (West Haven, CT, USA). For in vitro studies, sorafenib at various concentrations was dissolved in DMSO and then added to the cells in Dulbecco's modified Eagle's medium (DMEM) containing 5\% fetal bovine serum (FBS). Chloroquine, Bafilomycin A1, cycloheximide and WP1066 were purchased from Sigma (Deisenhofen, Germany). Antibodies for immunoblotting such as Akt1, anti-pERK-1/2 ERK2 were purchased from Santa Cruz Biotechnology (San Diego, CA, USA). SHP-1 antibody was purchased from Abcam (Cambridge, UK). Other antibodies such as Bcl-xl, Bik, p-STAT3 (Tyr705), STAT3, p-Akt (Ser473), p-Akt (Thr308), LC3, Mcl-1, myc-tag, Beclin 1, Atg5, Atg3, P62, TSC1, p-TSC2, TSC2,
p-mTOR (S2481), mTOR, p-S6, S6, p-4EBP1 and 4EBP1 were purchased from Cell Signaling (Danvers, MA, USA).

Cell culture and western blot analysis. The PLC/PRF/5 (PLC5), Sk-Hep-1, Hep3B and HepG2 cell lines were obtained from American Type Culture Collection (Manassas, VA, USA). The cells were maintained in DMEM supplemented with $10 \%$ FBS, 100 units/ml penicillin G, $100 \mu \mathrm{g} / \mathrm{ml}$ streptomycin sulfate and $25 \mu \mathrm{g} / \mathrm{ml}$ amphotericin B in a humidified incubator at $37^{\circ} \mathrm{C}$ in an atmosphere of $5 \% \mathrm{CO}_{2}$ in air. Lysates of $\mathrm{HCC}$ cells treated with drugs at the indicated concentrations for various periods of time will be prepared for immunoblotting of LC3, p-STAT3, STAT3, and so on. Western blot analysis was performed as previously reported. ${ }^{38}$

Autophagy analysis. The following three methods were used to assess drug-induced autophagic cell death: (1) western blot analysis of microtubuleassociated protein-1 light chain 3 (LC3 II) as described previously; ${ }^{16,39,40}$ (2) electron microscopy: samples were fixed with $2.5 \%$ glutaraldehyde solution buffer in PBS at $4{ }^{\circ} \mathrm{C}$ for $1 \mathrm{~h}$, postfixed in $1 \%$ osmium tetroxide solution at $4{ }^{\circ} \mathrm{C}$ for $3 \mathrm{~h}$, dehydrated in graded concentrations of ethanol and embedded in LR white resin. Ultrathin sections $(70 \mathrm{~nm})$ were examined with a JEOL JEM-1400EX electron microscope (JEOL Inc., Tokyo, Japan) at $120 \mathrm{Kv}$; (3) immunofluorescence of acridine orange: $\mathrm{HCC}$ cells were grown on coverslips. After being washed with PBS, cells were treated with $20 \mu \mathrm{M}$ sorafenib or $10 \mu \mathrm{M}$ SC-59 for $16 \mathrm{~h}$, fixed with ice-cold $4 \%$ paraformaldehyde for $30 \mathrm{~min}$ at room temperature, then stained with acridine orange $(5 \mu \mathrm{g} / \mathrm{ml})$ for $5 \mathrm{~min}$ at room temperature. The cells were examined under a Leica DM2500 fluorescence microscope (Leica Microsystems GmbH, Wetzlar, Germany)

Cell viability assay. HCC cells including PLC5 and SK-Hep1 were seeded in 96-well plate at a density of 5000 cells per well. The cells were treated with sorafenib $(20 \mu \mathrm{M})$ or SC-59 $(10 \mu \mathrm{M})$ with indicated condition including overexpression of Mcl-1, knockdown of Beclin 1 or co-treatment with Bafilomycin A1 or $\mathrm{CQ}$ for $16 \mathrm{~h}$. The cell viability was measured by MTT assay.

Annexin V/PI staining. PLC5 cells were treated with indicated dose for $48 \mathrm{~h}$ and collected for Annexin V/PI-double staining. The analysis of annexin V binding was carried out with the Annexin V-FITC according to the manufacturer's instructions (eBioscience, San Diego, CA, USA). Briefly, the cells were incubated with sorafenib or SC-59 for $48 \mathrm{~h}$ and then collected, washed twice with cold PBS, centrifuged at 1500 r.p.m. for $5 \mathrm{~min}$ and resuspended in $1 \times$ Annexin $\mathrm{V}$ binding buffer at a concentration of $10^{6}$ cells/ml. Annexin V-FITC $(5 \mu l)$ and PI $(25 \mu \mathrm{g} / \mathrm{ml})$ were added into cell extract and incubated for $15 \mathrm{~min}$ at room temperature in the dark. The stained sample were analyzed with Gallios Flow Cytometry (Beckman Coulter Inc., Fullerton, CA, USA) based on the manufacturer's instructions.

Real-time PCR. Total RNA was isolated from PLC5 with RNA extraction kit (Ambion-Invitrogen, Lennik, Belgium) and cDNA was prepared from $2.5 \mu \mathrm{g}$ RNA using SuperScript.

Vilo cDNA Synthesis Kit by the manufacturer's instructions (Invitrogen, Carlsbad, $\mathrm{CA}, \mathrm{USA}$ ). Oligonucleotide sequences were used as follows: GAPDH, $5^{\prime}-\mathrm{CGA}$ CCACTTTGTCAAGCTCA-3' (sense) and $5^{\prime}$-AGGGGTCTACATGGCAACTG- ${ }^{\prime}$ (anti-sense); Mcl-1: $5^{\prime}$-CTTGCCACTTGCTTTTCTGG-3' (sense) and 5'-CAAGGC ATGCTTCGGAAACT- $3^{\prime}$ (antisense). The SYBR Green PCR reagent was used for quantitative PCR by the manufacturer's instructions (ABI, Carlsbad, CA, USA).

Gene knockdown using siRNA. Smart pool siRNA reagents, including a control (D-001810-10), SHP-1 and Beclin 1 were all purchased from Dharmacon (Chicago, IL, USA). The procedure was as described previously. ${ }^{38,41}$

PLC5 with ectopic expression of STAT3 and Mcl-1. STAT3 CDNA (KIAA1524) and Mcl-1 was purchased from Addgene plasmid repository (http://www.addgene.org/). PLC5 cells with stable expression of STAT3 or transient expression of $\mathrm{Mcl}-1$ were then treated with drugs, harvested and processed for western blot analysis as described previously. ${ }^{42}$

Activity of Raf-1 and SHP-1. Tyrosine Phosphatase Assay Kit (R-22067) was used for SHP-1 activity assay (Molecular Probes, Invitrogen). The Raf-1 kinase cascade assay kit (Upstate-Millipore, Billerica, MA, USA) was used to 
examine the Raf- 1 kinase activity. Both the assays were conducted according to the manufacturer's instructions.

Xenograft tumor growth. Male NCr athymic nude mice (5-7 weeks of age) were obtained from the National Laboratory Animal Center (Taipei, Taiwan). All experimental procedures using these mice were performed in accordance with protocols approved by the Institutional Laboratory Animal Care and Use Committee of National Taiwan University. Each mouse was inoculated s.c. in the dorsal flank with $1 \times 10^{6}$ PLC5 cells suspended in $0.1 \mathrm{ml}$ of serum-free medium containing 50\% Matrigel (BD Biosciences, Bedford, MA, USA). When the tumors reached $200-300 \mathrm{~mm}^{3}$, mice received sorafenib tosylate $(20 \mathrm{mg} / \mathrm{kg})$ or SC-59 $(20 \mathrm{mg} / \mathrm{kg})$ p.o. once daily. Controls received vehicle. Tumors will be measured weekly using calipers and their volumes calculated using the following standard formula: width ${ }^{2} \times$ length $\times 0.52$.

Statistical analysis. Comparisons of mean values were performed using the independent samples $t$-test in SPSS for Windows 11.5 software (SPSS, Inc., Chicago, IL, USA).

\section{Conflict of Interest}

Dr. Ann-Lii Cheng serves as a consultant and a member of the speaker's bureau for Bayer-Schering. All other authors declare no conflict of interest.

Acknowledgements. This study was supported by grants, NTUH $101 \mathrm{P} 01$ from National Taiwan University Hospital (K-F C), NSC99-2314-B-002-017-MY2 (K-F C) and NSC 100-2325-B-002 -032 (K-F C) from the National Science Council, Taiwan.

1. Kao JH, Chen DS. Changing disease burden of hepatocellular carcinoma in the Far East and Southeast Asia. Liver Int 2005; 25: 696-703.

2. Wang SH, Yeh SH, Lin WH, Wang HY, Chen DS, Chen PJ. Identification of androgen response elements in the enhancer I of hepatitis $B$ virus: a mechanism for sex disparity in chronic hepatitis B. Hepatology 2009; 50: 1392-1402.

3. Kao JH, Chen PJ, Chen DS. Recent advances in the research of hepatitis B virus-related hepatocellular carcinoma: epidemiologic and molecular biological aspects. Adv Cancer Res 2010; 108: 21-72.

4. Yeh SH, Chen PJ. Gender disparity of hepatocellular carcinoma: the roles of sex hormones. Oncology 2010; 78: 172-179.

5. Chen PJ, Yeh SH, Liu WH, Lin CC, Huang HC, Chen $\mathrm{CL}$ et al. Androgen pathway stimulates MicroRNA-216a transcription to suppress the tumor suppressor in lung cancer-1 gene in early hepatocarcinogenesis. Hepatology 2012; 56: 632-643.

6. Forner A, Llovet JM, Bruix J. Chemoembolization for intermediate HCC: is there proof of survival benefit? J Hepatol 2012; 56: 984-986.

7. Bruix J, Llovet JM. HCC surveillance: who is the target population? Hepatology 2003; 37: 507-509.

8. Shen YC, Hsu C, Cheng AL. Molecular targeted therapy for advanced hepatocellular carcinoma: current status and future perspectives. J Gastroenterol 2010; 45: 794-807.

9. Llovet JM, Ricci S, Mazzaferro V, Hilgard P, Gane E, Blanc JF et al. Sorafenib in advanced hepatocellular carcinoma. N Engl J Med 2008; 359: 378-390.

10. Cheng AL, Kang YK, Chen Z, Tsao CJ, Qin S, Kim JS et al. Efficacy and safety of sorafenib in patients in the Asia-Pacific region with advanced hepatocellular carcinoma: a phase III randomised, double-blind, placebo-controlled trial. Lancet Oncol 2009; 10: 25-34.

11. Rosmorduc O, Desbois-Mouthon C. Targeting STAT3 in hepatocellular carcinoma: sorafenib again. J Hepatol 2011; 55: 957-959.

12. Tai WT, Cheng AL, Shiau CW, Huang HP, Huang JW, Chen PJ et al. Signal transducer and activator of transcription 3 is a major kinase-independent target of sorafenib in hepatocellular carcinoma. J Hepatol 2011; 55: 1041-1048.

13. Zhang Q, Wang HY, Marzec M, Raghunath PN, Nagasawa T, Wasik MA. STAT3- and DNA methyltransferase 1-mediated epigenetic silencing of SHP-1 tyrosine phosphatase tumor suppressor gene in malignant T lymphocytes. Proc Natl Acad Sci USA 2005; 102: 6948-6953.

14. Chen KF, Tai WT, Hsu CY, Huang JW, Liu CY, Chen PJ et al. Blockade of STAT3 activation by sorafenib derivatives through enhancing SHP-1 phosphatase activity. Eur $J$ Med Chem 2012; 55: 220-227.

15. Chen KF, Tai WT, Huang JW, Hsu CY, Chen WL, Cheng AL et al. Sorafenib derivatives induce apoptosis through inhibition of STAT3 independent of Raf. Eur J Med Chem 2011; 46: 2845-2851.

16. Mizushima N. Autophagy: process and function. Genes Dev 2007; 21: 2861-2873.

17. David R. Metabolism: keeping fit with autophagy. Nat Rev Mol Cell Biol 2012; 13: 136.

18. Kang R, Zeh HJ, Lotze MT, Tang D. The Beclin 1 network regulates autophagy and apoptosis. Cell Death Differ 2011; 18: 571-580.

19. Bergmann A. Autophagy and cell death: no longer at odds. Cell 2007; 131: 1032-1034.

20. Codogno P, Mehrpour M, Proikas-Cezanne T. Canonical and non-canonical autophagy: variations on a common theme of self-eating? Nat Rev Mol Cell Biol 2012; 13: 7-12.

21. Maiuri MC, Zalckvar E, Kimchi A, Kroemer G. Self-eating and self-killing: crosstalk between autophagy and apoptosis. Nat Rev Mol Cell Biol 2007; 8: 741-752.

22. Pattingre S, Tassa A, Qu X, Garuti R, Liang XH, Mizushima N et al. Bcl-2 antiapoptotic proteins inhibit Beclin 1-dependent autophagy. Cell 2005; 122: 927-939.

23. Germain M, Nguyen AP, Le Grand JN, Arbour N, Vanderluit JL, Park DS et al. MCL-1 is a stress sensor that regulates autophagy in a developmentally regulated manner. EMBO J 2011; 30: 395-407.

24. Liu L, Cao Y, Chen C, Zhang X, McNabola A, Wilkie D et al. Sorafenib blocks the RAF/MEK/ERK pathway, inhibits tumor angiogenesis, and induces tumor cell apoptosis in hepatocellular carcinoma model PLC/PRF/5. Cancer Res 2006; 66: 11851-11858.

25. Blechacz BR, Smoot RL, Bronk SF, Werneburg NW, Sirica AE, Gores GJ. Sorafenib inhibits signal transducer and activator of transcription-3 signaling in cholangiocarcinoma cells by activating the phosphatase shatterproof 2. Hepatology 2009; 50: 1861-1870.

26. Chen KF, Tai WT, Liu TH, Huang HP, Lin YC, Shiau CW et al. Sorafenib overcomes TRAlL resistance of hepatocellular carcinoma cells through the inhibition of STAT3. Clin Cancer Res 2010; 16: 5189-5199.

27. Marquez RT, Xu L. Bcl-2:Beclin 1 complex: multiple, mechanisms regulating autophagy/ apoptosis toggle switch. Am J Cancer Res 2012; 2: 214-221.

28. Shi YH, Ding ZB, Zhou J, Hui B, Shi GM, Ke AW et al. Targeting autophagy enhances sorafenib lethality for hepatocellular carcinoma via ER stress-related apoptosis. Autophagy 2011; 7: 1159-1172.

29. Shimizu S, Takehara T, Hikita H, Kodama T, Tsunematsu H, Miyagi T et al. Inhibition of autophagy potentiates the antitumor effect of the multikinase inhibitor sorafenib in hepatocellular carcinoma. Int J Cancer 2012; 131: 548-557.

30. Maiuri MC, Criollo A, Tasdemir E, Vicencio JM, Tajeddine N, Hickman JA et al. BH3-only proteins and $\mathrm{BH} 3$ mimetics induce autophagy by competitively disrupting the interaction between Beclin 1 and Bcl-2/Bcl-X(L). Autophagy 2007; 3: 374-376.

31. Maiuri MC, Criollo A, Kroemer G. Crosstalk between apoptosis and autophagy within the Beclin 1 interactome. EMBO J 2010; 29: 515-516.

32. Ku B, Woo JS, Liang $\mathrm{C}$, Lee $\mathrm{KH}$, Jung JU, Oh BH. An insight into the mechanistic role of Beclin 1 and its inhibition by prosurvival Bcl-2 family proteins. Autophagy 2008; 4: 519-520.

33. Maiuri MC, Le Toumelin G, Criollo A, Rain JC, Gautier F, Juin P et al. Functional and physical interaction between $\mathrm{Bcl}-\mathrm{X}(\mathrm{L})$ and a $\mathrm{BH}$-like domain in Beclin-1. EMBO J 2007; 26: 2527-2539.

34. Zalckvar E, Berissi H, Eisenstein M, Kimchi A. Phosphorylation of Beclin 1 by DAP-kinase promotes autophagy by weakening its interactions with Bcl-2 and Bcl-XL. Autophagy 2009; 5: 720-722.

35. Tang Y, Hamed HA, Cruickshanks N, Fisher PB, Grant S, Dent P. Obatoclax and lapatinib interact to induce toxic autophagy through NOXA. Mol Pharmacol 2012; 81: 527-540.

36. Yokoyama T, Kondo Y, Kondo S. Roles of mTOR and STAT3 in autophagy induced by telomere $3^{\prime}$ overhang-specific DNA oligonucleotides. Autophagy 2007; 3: 496-498.

37. Yoon S, Woo SU, Kang JH, Kim K, Kwon MH, Park S et al. STAT3 transcriptional factor activated by reactive oxygen species induces IL 6 in starvation-induced autophagy of cancer cells. Autophagy 2010; 6: 1125-1138.

38. Chen KF, Yeh PY, Yeh KH, Lu YS, Huang SY, Cheng AL. Down-regulation of phospho-Akt is a major molecular determinant of bortezomib-induced apoptosis in hepatocellular carcinoma cells. Cancer Res 2008; 68: 6698-6707.

39. Itakura E, Mizushima N. p62 Targeting to the autophagosome formation site requires selfoligomerization but not LC3 binding. J Cell Biol 2011; 192: 17-27.

40. Tsujimoto Y, Shimizu S. Another way to die: autophagic programmed cell death. Cell Death Differ 2005; 12(Suppl 2): 1528-1534.

41. Tai WT, Cheng AL, Shiau CW, Liu CY, Ko CH, Lin MW et al. Dovitinib induces apoptosis and overcomes sorafenib resistance in hepatocellular carcinoma through SHP-1-mediated inhibition of STAT3. Mol Cancer Ther 2012; 11: 452-463.

42. Chen KF, Yeh PY, Hsu C, Hsu CH, Lu YS, Hsieh HP et al. Bortezomib overcomes tumor necrosis factor-related apoptosis-inducing ligand resistance in hepatocellular carcinoma cells in part through the inhibition of the phosphatidylinositol 3-kinase/Akt pathway. J Biol Chem 2009; 284: 11121-11133.

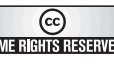

Cell Death and Disease is an open-access journal published by Nature Publishing Group. This work is licensed under the Creative Commons Attribution-NonCommercial-No Derivative Works 3.0 Unported License. To view a copy of this license, visit http://creativecommons.org/licenses/by-nc-nd/3.0/ 